\title{
Commentary: Heat stress-induced neuroinflammation and aber- ration in monoamine levels in hypothalamus are associated with temperature dysregulation
}

\author{
Nishant Ranjan Chauhan', Rajinder Kumar Gupta', Shashi Bala Singh²* \\ 'Defence Institute of Physiology and Allied Sciences (DIPAS), Defence Research and Development Organisation (DRDO), Lucknow Road, Timarpur, Delhi \\ 110054, India \\ 2Distinguished Scientist and Director General (Life Sciences), Defence Research and Development Organisation (DRDO), DRDO Bhawan, Rajaji Marg, Delhi \\ 110011, India
}

\section{Article Info}

\section{Article Notes}

Received: August 01, 2017

Accepted: October 10, 2017

\section{*Correspondence:}

Dr. Shashi Bala Singh

Distinguished Scientist and Director General (Life Sciences), Defence Research and Development Organization (DRDO),

DRDO Bhawan, Rajaji Marg, Delhi 110011, INDIA

Tel: +91 1123016976

Fax: +91 11 23014259, Email: sbsdrdo@gmail.com

Email: drshashisingh@gmail.com

C 2017 Singh SB. This article is distributed under the terms of the Creative Commons Attribution 4.0 International License

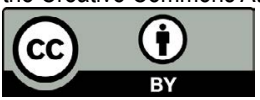

Keywords

Hypothalamus

Chronic inflammation

Heat stroke

Monoamines

Glutamate

Thermoregulatory disruption

\section{ABSTRACT}

Heat stress (HS) is a common stressor that affects all biological systems. Mild to moderate HS is associated with intact baroreflex response which tries to cope up with the stress by maintaining mean arterial pressure (MAP). However, during severe HS, baroreflex response fails leading to fall in MAP which is a pathognomonic feature of heat stroke. Heat stroke can induce neuroinflammation, brain ischemia, oxidative stress and neuronal damage. Increase in ambient temperature led to activation of the thermoregulatory process in Hypothalamus (HTH) and was achieved by rise in nor-epinephrine and fall in serotonin, whereas neurotransmitter imbalance occurred during severe HS in $\mathrm{HTH}$ and was associated with expression of inflammatory mediators. Results of our preliminary study also suggested that neuroinflammation was associated with neurotransmitter (monoamines and glutamate) imbalance in HTH leading to thermoregulatory disruption during severe HS. Here, we also discussed that individuals predisposed to factors like chronic inflammation and other complications could decrease the threshold of heat tolerance since a short episode of even sub maximal heat exposure would precipitate the inflammatory cascade leading to thermoregulatory shutdown.

\section{Heat Stroke}

Exposure to high ambient temperature initiates wide physiological responses like sending afferent signals from peripheral thermoreceptors to the preoptic anterior hypothalamus (POAH) followed by efferent signals from CNS to the periphery. It results in an increase in sympathetic activity and peripheral vasodilation enabling intravascular fluid to take away the heat from core to periphery and cool down the skin by evaporation. However, HS related maladies result when the compensatory mechanisms are exhausted either due to overexposure to heat or predisposing factors that increase susceptibility to heat intolerance. In its severe form, patients cease to sweat and core temperature rises $>41^{\circ} \mathrm{C}$ along with ataxia and altered sensorium which is a pathological condition termed as heat stroke. Uncompensated heat stroke is characterized by hyperthermia associated with the systemic inflammatory response that leads to multi organ dysfunction syndrome (MODS), in which central nervous system (CNS) dysfunctions prevail ${ }^{1}$. Heat stroke related deaths have increased worldwide as extreme weather events are the consequences of anthropogenic climatic changes $^{2-5}$. Cases of dehydration, low physical fitness, obesity, lack of 
acclimatization, drug abuse, diabetes, chronic obstructive pulmonary disease (COPD), cardiovascular insufficiency, skin disorders, malignant hyperthermia should be regarded a priority as predisposing factors for heat intolerance ${ }^{6-8} .30 \%$ of heat stroke survivors experience disability and neurological dysfunction even after cooling of the entire body ${ }^{9-11}$. It is inevitable that brain ischemia, inflammation and neuronal damage are the main causes of heat stroke, rather than body hyperthermia ${ }^{12-15}$. Generally, exertional heat stroke occurs in active healthy individuals during strenuous activity under HS conditions while nonexertional heat stroke occurs in elderly, COPD, chronic diabetics or immunocompromised individuals having systemic inflammation? ${ }^{7}$.

\section{Chronic Inflammation and Heat Susceptibility}

Chronic inflammation in diabetes causes autonomic insufficiency due to central and peripheral neuropathy thus, individuals at risk may not show cardinal symptoms of heat stroke $^{16,17}$. al-Harthi et al reported that most of the patients with heat stroke at Mecca Pilgrimage had diabetes with hyperglycemia, suggesting a strong correlation of chronic inflammation with heat susceptibility ${ }^{18}$. Also, patients with chronic inflammatory encephalopathy characterized by oxidative and neuronal damage, ischemic injury and brain inflammation ${ }^{19-21}$ are more prone to heat intolerance. Hyperglycemia is known to aggravate brain damage by inducing an ischemic stroke in experimental animals ${ }^{22}$. The detrimental effect of hyperglycemia on cerebral ischemia is considered to be mediated by the overproduction of glutamate, free radicals, and lactic acid ${ }^{23,24}$. Michel et al have proposed that decreased heat tolerance is associated with hypothalamic-pituitary-adrenal (HPA) axis impairment ${ }^{25}$. Hyperglycemic or diabetic animals could exacerbate the hypothalamic neuronal damage and dysfunction of the HPA axis mechanism and resulted in more severe multiple organ failure during heat stroke ${ }^{26}$. Excessive elevation of glutamate, lactate/pyruvate ratio and glycerol in the hypothalamus of diabetic rats occur after heat stroke onset $^{26}$. Studies suggested that chronic inflammation in diabetes can induce functional and structural plasticity in neurons, including synaptic vesicle reduction in mossy fiber nerve terminals ${ }^{27}$. Evidence also demonstrated that chronic inflammation induces alteration in neurotransmitter release in different brain areas. Neurotransmitters and various brain regions emerge to be differentially affected, and the effects also depend on the duration and severity of diabetes ${ }^{28-33}$. Published report indicated that chronic inflammation reduces the activity of the nigrostriatal dopaminergic system ${ }^{34}$. The alterations in neurotransmitter release caused by chronic inflammation might result, partially, from changes in the exocytotic machinery ${ }^{35}$, since reduced release from terminal vesicles causes a decline in catecholamines synthesis with increased storage ${ }^{36}$.
All the above studies give an insight into the cellular and molecular mechanisms of the neurotransmitter release under chronic inflammatory conditions, thereby disrupting thermal homeostasis of the individual. The previous study has shown that administration of hyperbaric oxygen in diabetic rats after heat stroke exhibited attenuating brain inflammation, ischemia, neuronal and oxidative damage ${ }^{26}$.

\section{Relevance of the Present Study}

Although ample data showed that chronic inflammation primes the brain tissue for acute HS damage thus reducing thermotolerance but sparse literature is available on the correlation of acute inflammation with thermoregulation at HTH during the progressive increase in HS. There was also a necessity of simultaneous comparison of neurotransmitter release in HTH and systemic biochemical changes with an increase in the severity of HS. Therefore, our study extensively evaluated the biochemical parameters and provided an insight into the monoamine and glutamate release in both HTH as well as in systemic circulation during HS. We correlated the biochemical findings along with the molecular \& physiological parameters and observed that severity of inflammation was associated with neurotransmitter imbalance during the acute thermal insult. However, our study has some limitations as the recording of the cerebral blood flow (CBF) would have given better information about the cerebral ischemia during heat exposure. Behavioural studies associated with cognition and memory functions of the brain and its correlation with biochemical changes in HTH with increasing severity of HS would have provided a better biological understanding of thermoregulation.

Although our data ratifies that during moderate HS, systemic circulation showed an increase in levels of stress hormones while HTH showed normal neurotransmitter balance and thermal regulation with no evidence of inflammation. This suggests that hypothalamic tissue (neurons) is comparatively resistant to ischemia ${ }^{37}$ which makes it to perform its functions even during the progressive increase in HS conditions. This is quite interesting and unwraps the new avenues to work upon the unexplored mechanisms behind it. Understanding how neurons naturally protect against ischemia will help to identify molecular targets in the brain to improve survival of the susceptible areas following heat stroke. Therefore, it might be relevant to suggest the prophylactic role of anti-inflammatory agents during peak summers in heat susceptible individuals since a short episode of even sub maximal threshold heat exposure can precipitate the inflammatory cascade leading to thermoregulatory shutdown.

\section{Acknowledgement}

This study was financially supported by Defence 
Research and Development Organization (DRDO), Ministry of Defence, Govt. of India and our sincere thanks to Director, Defence Institute of Physiology and Allied Sciences (DIPAS) for help and support.

\section{Conflict of interest}

The authors declare that there is no conflict of interest.

\section{References}

1. Bouchama A, Knochel JP. Heat stroke. N Engl J Med. 2002; 346: 19781988.

2. Mann ME, Rahmstorf S, Kornhuber K, et al. Influence of Anthropogenic Climate Change on Planetary Wave Resonance and Extreme Weather Events. Sci Rep. 2017; 7: 45242.

3. Petoukhov V, Rahmstorf S, Petri S, et al. Quasiresonant amplification of planetary waves and recent Northern Hemisphere weather extremes. Proc Natl Acad Sci U S A. 2013; 110: 5336-5341.

4. Horton RM, Mankin JS, Lesk C, et al. A Review of Recent Advances in Research on Extreme Heat Events. Current Climate Change Reports. 2016; 2: 242-259.

5. Li T, Ding F, Sun Q et al. Heat stroke internet searches can be a new heatwave health warning surveillance indicator. Sci Rep. 2016; 6: 37294.

6. Leon LR, Bouchama A. Heat stroke. Compr Physiol. 2015; 5: 611-647.

7. Chen SH, Lin MT, Chang CP. Ischemic and oxidative damage to the hypothalamus may be responsible for heat stroke. Curr Neuropharmacol. 2013; 11: 129-140.

8. Epstein Y. Heat intolerance: predisposing factor or residual injury. Med Sci Sports Exerc. 1990; 22: 29-35.

9. Dematte JE, O’Mara K, Buescher J, et al. Near-fatal heat stroke during the 1995 heat wave in Chicago. Ann Intern Med. 1998; 129: 173-181.

10. Argaud L, Ferry T, Le QH, et al. Short- and long-term outcomes of heatstroke following the 2003 heat wave in Lyon, France. Arch Intern Med. 2007; 167: 2177-2183.

11. Wallace RF, Kriebel D, Punnett L, et al. Prior heat illness hospitalization and risk of early death. Environ Res. 2007; 104: 290-295.

12. Shih CJ, Lin MT, Tsai SH. Experimental study on the pathogenesis of heat stroke. J Neurosurg. 1984; 60: 1246-1252.

13. Chen SH, Niu KC, Lin MT. Cerebrovascular dysfunction is an attractive target for therapy in heat stroke. Clin Exp Pharmacol Physiol. 2006; 33: 663-672.

14. Shen $\mathrm{KH}$, Chang CK, Lin MT, et al. Interleukin-1 receptor antagonist restores homeostatic function and limits multiorgan damage in heatstroke. Eur J Appl Physiol. 2008; 103: 561-568.

15. Yang TH, Shih MF, Wen YS, et al. Attenuation of circulatory shock and cerebral ischemia injury in heat stroke by combination treatment with dexamethasone and hydroxyethyl starch. Exp Transl Stroke Med. 2010; $2: 19$.

16. Martyn CN, Hughes RA. Epidemiology of peripheral neuropathy. J Neurol Neurosurg Psychiatry. 1997; 62: 310-318.

17. Cyrus J, Broadstone VL, Pfeifer MA, et al. Diabetic peripheral neuropathy. Part II. Autonomic neuropathies (continuing education credit). Diabetes Educ. 1987; 13: 111-115.

18. al-Harthi SS, Karrar O, al-Mashhadani SA, et al. Metabolite and hormonal profiles in heat stroke patients at Mecca pilgrimage. J Intern Med. 1990; 228: 343-346.

19. Mastrocola R, Restivo F, Vercellinatto I, et al. Oxidative and nitrosative stress in brain mitochondria of diabetic rats. J Endocrinol. 2005; 187 : 37-44.

20. Shukla V, Shakya AK, Perez-Pinzon MA, et al. Cerebral ischemic damage in diabetes: an inflammatory perspective. J Neuroinflammation. 2017; 14: 21.

21. Zhang Z, Yan J, Shi H. Hyperglycemia as a Risk Factor of Ischemic Stroke. J Drug Metab Toxicol. 2013; 4.

22. Demchuk AM, Morgenstern LB, Krieger DW, et al. Serum glucose level and diabetes predict tissue plasminogen activator-related intracerebral hemorrhage in acute ischemic stroke. Stroke. 1999; 30: 34-39.

23. Li PA, Siesjo BK. Role of hyperglycaemia-related acidosis in ischaemic brain damage. Acta Physiol Scand. 1997; 161: 567-580.

24. Wei J, Quast MJ. Effect of nitric oxide synthase inhibitor on a hyperglycemic rat model of reversible focal ischemia: detection of excitatory amino acids release and hydroxyl radical formation. Brain Res. 1998; 791: 146-156.

25. Michel V, Peinnequin A, Alonso A, et al. Decreased heat tolerance is associated with hypothalamo-pituitary-adrenocortical axis impairment. Neuroscience. 2007; 147: 522-531.

26. Lee KL, Niu KC, Lin MT, et al. Attenuating brain inflammation, ischemia, and oxidative damage by hyperbaric oxygen in diabetic rats after heat stroke. J Formos Med Assoc. 2013; 112: 454-462.

27. Magarinos AM, McEwen BS. Experimental diabetes in rats causes hippocampal dendritic and synaptic reorganization and increased glucocorticoid reactivity to stress. Proc Natl Acad Sci U S A. 2000; 97: 11056-11061.

28. Guyot LL, Diaz FG, O'Regan MH, et al. The effect of streptozotocininduced diabetes on the release of excitotoxic and other amino acids from the ischemic rat cerebral cortex. Neurosurgery. 2001; 48: 385390; discussion 390-381.

29. Morris MJ, Pavia JM. Increased endogenous noradrenaline and neuropeptide Y release from the hypothalamus of streptozotocin diabetic rats. Brain Res. 2004; 1006: 100-106.

30. Yamato T, Misumi Y, Yamasaki S, et al. Diabetes mellitus decreases hippocampal release of neurotransmitters: an in vivo microdialysis study of awake, freely moving rats. Diabetes Nutr Metab. 2004; 17: 128-136.

31. Miyata S, Yamada N, Hirano S, et al. Diabetes attenuates psychological stress-elicited 5-HT secretion in the prefrontal cortex but not in the amygdala of mice. Brain Res. 2007; 1147: 233-239.

32. Misumi Y, Yamato T, Obata T, et al. Effects of ion channel blockers on basal hippocampal monoamine levels in freely moving diabetic and non-diabetic rats. Int J Neurosci. 2008; 118: 761-780.

33. Satoh E, Takahashi A. Experimental diabetes enhances $\mathrm{Ca} 2+$ mobilization and glutamate exocytosis in cerebral synaptosomes from mice. Diabetes Res Clin Prac. 2008; 81: e14-17.

34. Gallego M, Setien R, Izquierdo MJ, et al. Diabetes-induced biochemical changes in central and peripheral catecholaminergic systems. Physiol Res. 2003; 52: 735-741.

35. Gaspar JM, Baptista FI, Galvao J, et al. Diabetes differentially affects the content of exocytotic proteins in hippocampal and retinal nerve terminals. Neuroscience. 2010; 169: 1589-1600.

36. Gallego M, Espina L, Casis O. Blood pressure responsiveness to sympathetic agonists in anaesthetised diabetic rats. J Physiol Biochem. 2002; 58: 87-93.

37. Brisson CD, Lukewich MK, Andrew RD. A distinct boundary between the higher brain's susceptibility to ischemia and the lower brain's resistance. PLoS One. 2013; 8: e79589. 